\title{
The Assist Curve Design for Electric Power Steering System
}

\author{
Qinghe $\mathrm{Liu}^{1, \mathrm{a}}$, Weiguang Kong ${ }^{2, \mathrm{~b}}$ and Tao $\mathrm{Li}^{3, \mathrm{c}}$ \\ 1,2,3 Harbin Institute of Technology, Weihai 264209, China \\ a70872879@qq.com, bautokwg04@126.com, 'HIT_LITAO@163.com
}

Keywords: EPS; New type assist characteristic curve; Steering portability; Road feel.

\begin{abstract}
Three kinds of power curve are analyzed aimed at the sense of strength and steering portability. Thus, we put forward a new design principle based on the existing research. The assist characteristic range is divided into four kinds according to the vehicle speed and steering wheel torque. The simulation model of Electric Power Steering system is established in MATLAB/Simulink. The simulation results show that the new type of power assisted characteristic curve can better meet the steering portability during the low speed and good road feel at the high speed. Besides, the new type power assisted characteristic curve can avoid the frequent starting of motor and can protect the motor.
\end{abstract}

\section{Introduction}

Electric Power Steering system (EPS) is an advanced technology in line with the theme of energy saving, environmental protection and safety of automobiles, of course, EPS is the trend of automobile steering system [1]. The power assist characteristic is one of the core technology of the EPS, which determines the value of the assistance torque under certain speed and steering wheel torque. Also, the curve is the basis of the entire EPS work. From the existing research, because of the impact of the light and the intensity of the steering wheel, there is no accurate and detailed power torque and speed and steering wheel torque function relationship. By analyzing the previous research results, we will ensure the portability and road feel and according to the different speed range and steering wheel torque range, a new type of power assist characteristic curve is designed.

\section{Analysis and design of power assist characteristic curve}

Analysis of common power assist characteristic curve. The power assist characteristic curve is a function curve of steering torque on the speed and steering wheel torque. There are three types of power assist characteristic curve, such as the straight line, fold line and curve type [2]. By analyzing the three curves in term of the steering portability and road feel, we can find that curve type assist characteristic is better than the fold line and fold line type is better than that of linear from the road feel, while, linear model is superior to the fold line type, line type is better than that of curve type from the steering portability. From the above analysis and taking into account the characteristics of the motor itself and the jitter of the steering wheel, this paper presents a new type of characteristic curve. The design principle of the power assist characteristic curve is following [3]: (1) when steering wheel torque is in a small range between the zero, the motor provides small power or no power, while the input torque is large and the vehicle speed is low, it is needed to provide a larger torque; (2) The transition among different regions should be smooth to prevent the phenomenon of power jitter caused by the curve mutation.

Design of new type power assist characteristic curve. Combined with the design principle of the assist characteristic curve, this paper presents a new type assist characteristic curve, which is divided into four intervals including, low-speed small steering wheel torque area, low-speed large steering wheel torque area, high-speed small steering wheel torque area, high-speed large steering wheel torque area.

According to the speed definition on the power characteristics by Volkswagen, the speed of $0-50 \mathrm{~km} / \mathrm{h}$ is defined as the low speed range and $50-100 \mathrm{~km} / \mathrm{h}$ is defined as the speed range. In this 
paper, because the steering wheel torque maximum is $8 \mathrm{Nm}$, ruled by national standards, we define the steering wheel torque less than $4 \mathrm{Nm}$ as small area and greater than $4 \mathrm{Nm}$ as large area. Because the assist characteristic is a binary function of the vehicle speed and the steering torque, the characteristics of the binary function can be decomposed into a function of vehicle speed $K(v)$ and a basic power function of steering wheel torque $f\left(T_{d}\right)$. In this paper, the two functions are designed respectively. The expression of the function is as Eq. 1.

$$
T_{a}=\left\{\begin{array}{lr}
0 & 0 \leq T_{d}<T_{d 0} ; \\
K(v) * f_{1}\left(T_{d}\right) & T_{d 0} \leq T_{d}<T_{d 1} ; \\
K(v) * f_{2}\left(T_{d}\right) & T_{d 1} \leq T_{d}<T_{d \max } ; \\
T_{a \max } & T_{d} \geq T_{d \max } ;
\end{array}\right.
$$

Significance of parameters: $T_{d}$ means steering wheel torque; $T_{d 0}$ means steering wheel torque at the starting of motor; $T_{\text {dmax }}$ means maximum steering wheel torque; $T_{a}$ means power assist torque; $f_{1}\left(T_{d}\right), f_{2}\left(T_{d}\right)$ means different basic power function forms.

According to the corresponding datas between the speed and $K(v), K(v)$ is fitted through the form of the exponential function.

The design of the basic power function $f\left(T_{d}\right)$ requires a lot of parameters. The situ steering resistance torque can be measured by the test and can also be calculated by empirical formula [4]. In this paper, the maximum resistance torque is fixed to $381.62 \mathrm{Nm}$ by empirical formula, and then through the conversion to obtain the maximum torque $3.1 \mathrm{Nm}$.

At the speed $0-50 \mathrm{~km} / \mathrm{h}$ low speed range, the basic power function is designed based on the torque range of steering torque. In the small steering wheel torque region, the basic power assist function is designed as the quadratic function curve, which is a concave curve. At this time, the power motor is not very sensitive to the steering wheel torque changes, which makes the motor start and stop not frequent. Meanwhile, there is conducive to the protection of the motor and can also satisfy the power demand. When the steering torque is large, the power assist function is three times function curve, a convex curve. In low speed and high torque range, it needs larger assist torque to keep steering portability and concave curve can provide larger torque compared with the liner curve.

At the high speed range $50-100 \mathrm{~km} / \mathrm{h}$, the basic power function is also designed based on the torque range of steering torque. In small steering wheel torque, we still design basic power function for the quadratic function curve while in large steering wheel torque region, considering the high requirements of the road feel, we design basic power function as a straight line.

Above all, this paper design the new type power assist characteristic curve is shown in Fig. 1. 


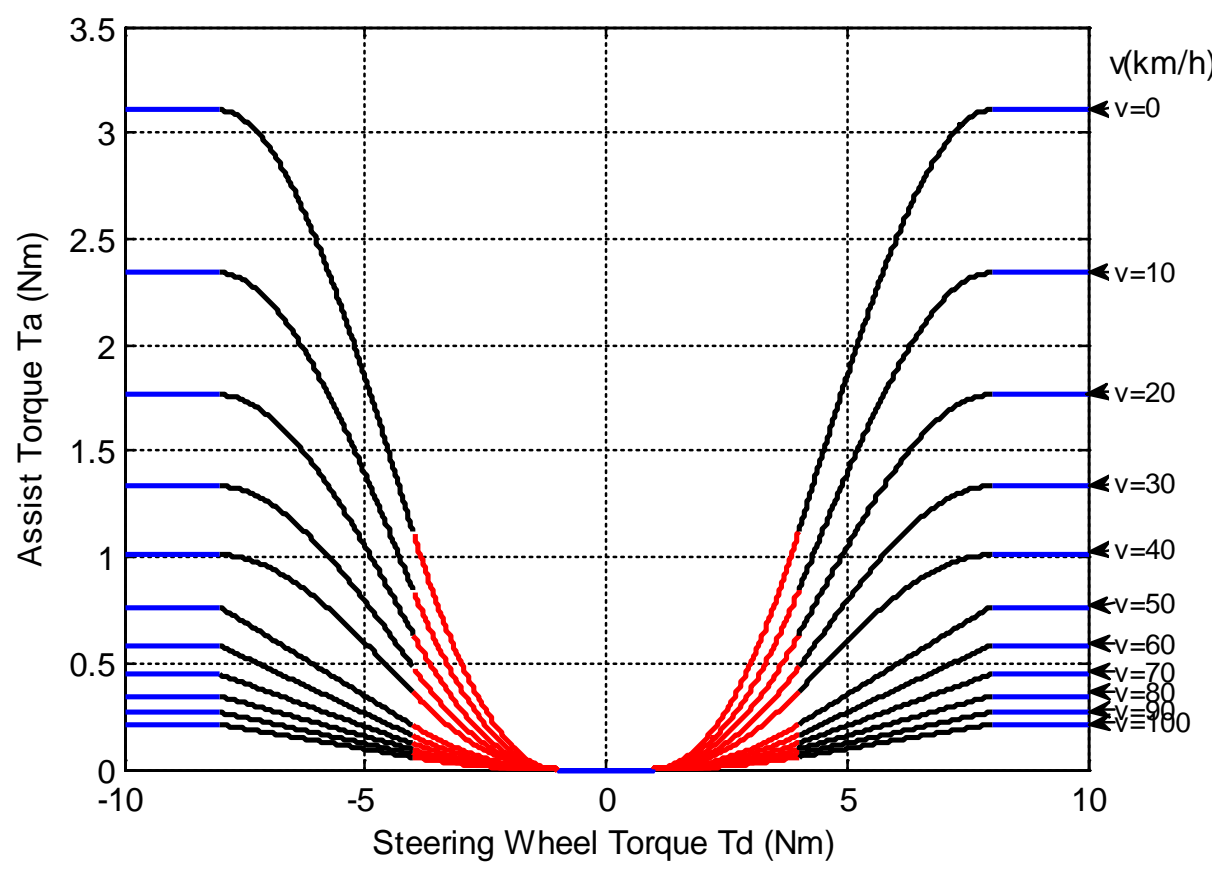

Fig. 1 New type power assist characteristic curve

\section{Model simulating and analysis}

In order to verify the reasonableness of the new power curve, it is necessary to develop an electric power steering system simulation model. EPS composed by the original mechanical steering mechanism and control section [5]. According to the mathematical model and conduct current PID control, we establish the corresponding whole simulation model, as shown in Fig. 2. In the model, input is speed and steering wheel torque and output is assist torque.

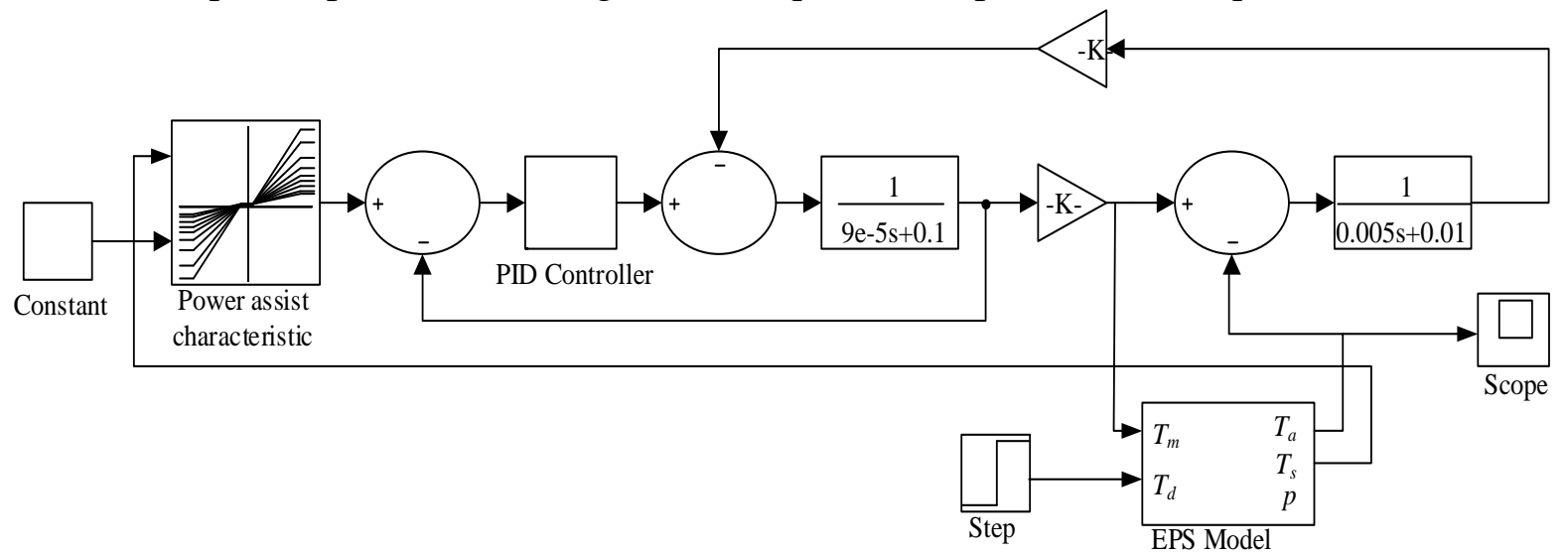

Fig. 2 Whole EPS simulation model

This article will compare the assist torque for each new curves and linear power curve. Fig. 3 shows the vehicle speed is $30 \mathrm{~km} / \mathrm{h}$ and the steering wheel torque is $3 \mathrm{Nm}$. Fig. 4 shows the vehicle speed is $30 \mathrm{~km} / \mathrm{h}$ and the steering wheel torque is $8 \mathrm{Nm}$. Fig. 5 shows the vehicle speed is $70 \mathrm{~km} / \mathrm{h}$ and the steering wheel torque is $3 \mathrm{Nm}$. Fig. 6 shows the vehicle speed is $70 \mathrm{~km} / \mathrm{h}$ and the steering wheel torque is $8 \mathrm{Nm}$. 


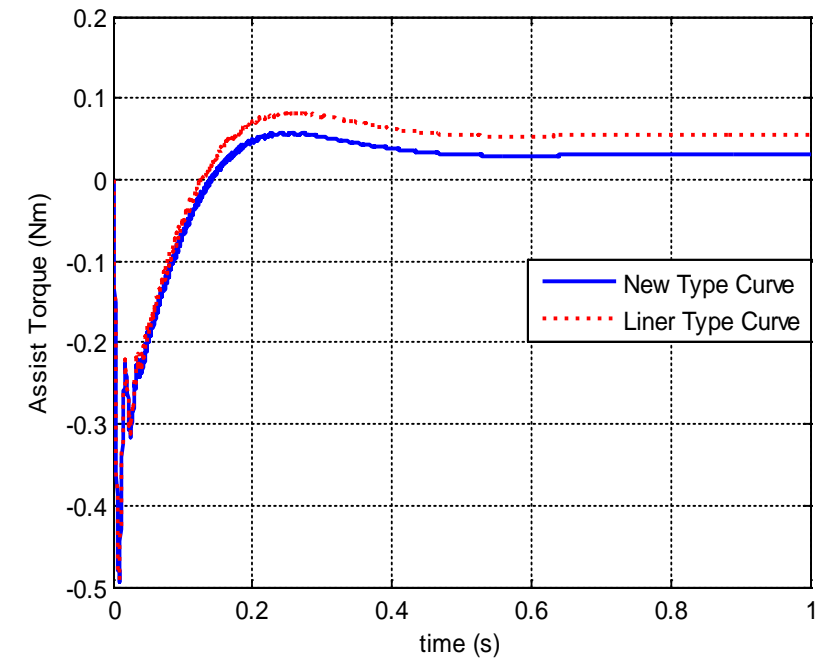

Fig. 3 Assist torque

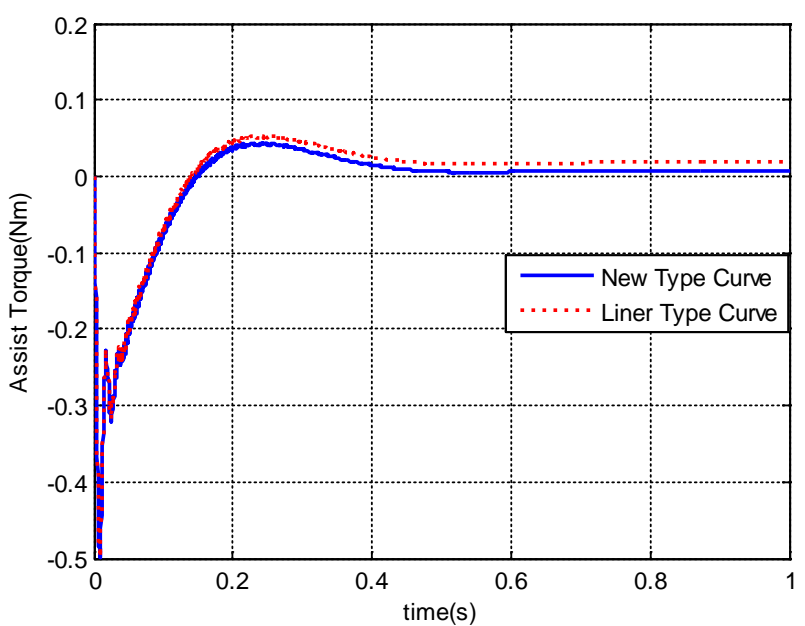

Fig. 5 Assist torque

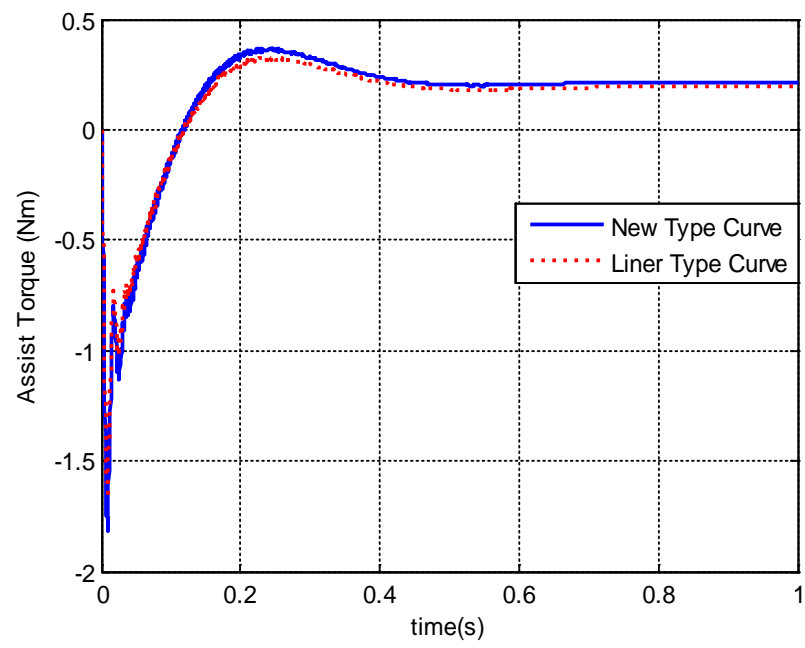

Fig. 4 Assist torque

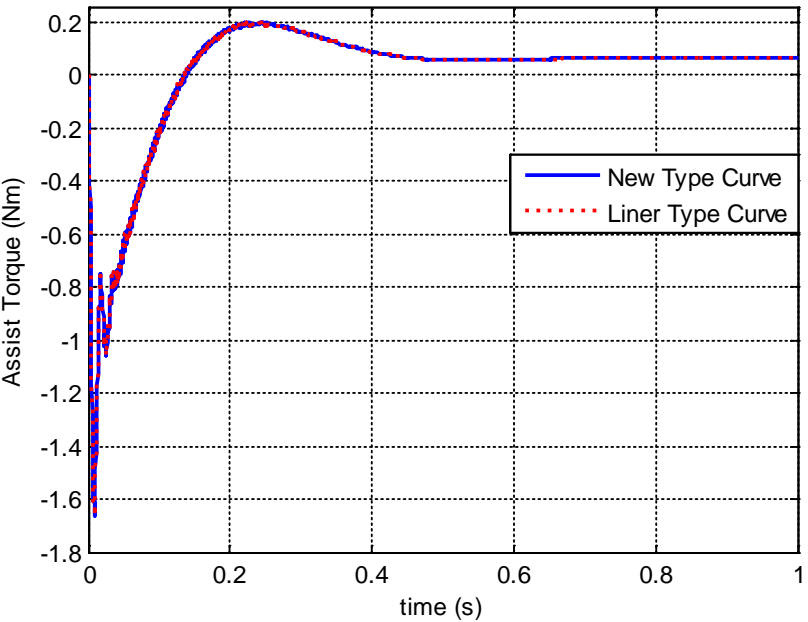

Fig. 6 Assist torque

As can be seen from Fig. 3, at low speed and small steering wheel torque, EPS with new assist characteristic curve can provide smaller assist torque. This will avoid frequent start of the motor and protect motor. As can be seen from Fig. 4, EPS with new assist characteristic curve provide greater assist torque and this means that EPS with new assist characteristic curve lighter and faster response. As can be seen from Fig. 5, at high speed and small steering wheel torque, under the same conditions of the steering wheel speed and vehicle speed, EPS with new assist characteristic curve provide smaller assist torque. This will avoid frequent start the motor and protect motor and ensure the strength of road feel at high speed. As can be seen from Fig. 6, EPS with linear power curve can provide a slightly larger assist torque. This means that the new curve have good road feel in the high-speed and large steering wheel torque .

\section{Summary}

In this paper, we put forward a new design principle based on the existing research. The assistance characteristic range is divided into four kinds according to the vehicle speed and steering wheel torque and simulate model of Electric Power Steering system (EPS) is established in MATLAB/Simulink. Besides, we simulate the EPS model under the straight line curve and the new type curve. The simulation results show that the new type of power assisted characteristic curve can better meet the steering portability during the low speed and good road feel at the high speed. Meanwhile, the new type power assist characteristic curve can protect the motor. In a word, the design is reasonable and effective. 


\section{Acknowledgement}

The authors would like to thank the financial support of Shandong science and technology research program project (2014GGX105006).

\section{References}

[1] L.N. Tian, W.T. Yang, Study on the effect of EPS control parameters of the system operation stability of vehicle transient. Machine China. 2015(17):112-113.

[2] Y.C. Wang, Research on control strategy of electric power steering system. Journal of Transport Information and Safety, 2005, 23(6):75-78.

[3] Badaway. A., Zuraski, J., Modeling and analysis of an electric power steering system. Automotive Engineering. 1998(1069), 25-31.

[4] Y.X. Zhao, Y.C. Zhu, Calculation and comparison of the empirical formula of vehicle turning resistance moment. Automobile Technology, 2015(7):102-103.

[5] G.B. Shi, Research on Simulation and control strategy of electric power steering system, Ji Lin University. 